\title{
Comment a-t-on traité la littérature française dans les lycées et collèges allemands entre 1850 et 1900 ?
}

Herbert Christ

\section{CpenEdition}

\section{Journals}

Electronic version

URL: https://journals.openedition.org/dhfles/3050

DOI: $10.4000 /$ dhfles.3050

ISSN: 2221-4038

Publisher

Société Internationale pour l'Histoire du Français Langue Étrangère ou Seconde

\section{Printed version}

Date of publication: 1 June 1999

Number of pages: p.248-270

ISSN: 0992-7654

\section{Electronic reference}

Herbert Christ, "Comment a-t-on traité la littérature française dans les lycées et collèges allemands entre 1850 et 1900?", Documents pour l'histoire du français langue étrangère ou seconde [Online], 23 | 1999, Online since 02 June 2015, connection on 10 March 2023. URL: http://journals.openedition.org/ dhfles/3050 ; DOI: https://doi.org/10.4000/dhfles.3050

This text was automatically generated on 10 March 2023.

All rights reserved 


\title{
Comment a-t-on traité la littérature française dans les lycées et collèges allemands entre 1850 et 1900 ?
}

\author{
Herbert Christ
}

\section{Remarques préliminaires}

1 Cette étude part d'un point de vue méthodologique : Comment les professeurs de français de la seconde moitié du XIX ${ }^{e}$ siècle ont-ils enseigné la littérature ? Pour avoir une réponse, il faudra leur donner la parole. On ne dispose pas de rapports d'inspection, de peu de témoignages d'élèves, les recherches empiriques contemporaines manquent. Les seules sources sont les témoignages publiés par les professeurs eux-mêmes. Comme correctif on dispose des programmes officiels, qui s'occupent occasionnellement de questions méthodologiques.

2 Les sources sont abondantes grâce à une tradition des lycées et collèges allemands, autrichiens, hongrois, suisses et même Scandinaves et russes, qui publiaient et distribuaient chaque année un " programme de l'établissement " - en allemand " Schulprogramm » - et qui contenait un rapport moral de la vie de l'établissement (y compris des statistiques, la liste des professeurs, des élèves, etc.), et en plus une contribution thématique d'un professeur de l'établissement. Ces contributions pouvaient - mais ne devaient pas - traiter des sujets didactiques.

3 J'ai dépouillé dans la bibliographie de ces Schulprogramme (Kössler 1987-1991) pour la période entre 1850 et 1900 à peu près 500 contributions concernant la littérature française. Il y en a parmi elles certaines qui peuvent nous donner une réponse à la question : Y avait-il, dans les pays de langue allemande, dans la deuxième moitié $d u$ XIX ${ }^{e}$ siècle des débuts de méthodologie(s) de la classe de littérature française? 


\section{La chrestomathie ou les auteurs}

Il serait erroné de croire que les problèmes méthodologiques aient occupé la première place dans les témoignages des professeurs. Très souvent ils s'en occupent en appendice après avoir longuement discuté du contenu de la classe de littérature, de ses finalités et du choix des textes littéraires. Mais même dans ces discussions didactiques au sens large du terme il y avait une question qui les occupait et qui peut être considérée comme le début d'une réflexion méthodologique : la question de savoir s'il faut travailler sur la base d'une chrestomathie ou de textes littéraires entiers (ou éventuellement abrégés pour l'usage des classes).

Rodovicz (1852) résume l'état de la discussion comme suit :

Quant au choix des auteurs et pour l'étendue des pièces qui devaient servir à la lecture, les professeurs de langue se sont divisés en deux camps opposés, entre lesquels on distinguerait encore ceux du bon juste milieu. Les uns préfèrent les pièces en entier ; ils lisent, du moins dans les classes supérieures, d'un bout à l'autre, quelques traités touchant l'histoire, la morale, la philosophie, quelques peintures poétiques en vers ou en prose, et principalement quelques drames, tant de l'école dite classique que du temps moderne. (p. 4).

$6 \quad$ Et il constate pour le parti opposé :

Les autres maîtres de langue (sic !), peut-être la majeure partie, n'hésitent point de blâmer grandement cette allure, en applaudissant la maxime suivant laquelle on a, de toute ancienneté, accordé la préférence aux chrestomathies, aux anthologies, ces bons recueils des plus beaux morceaux, mûrement choisis dans l'intention d'écarter des jeunes âmes sensibles tout ce qui pût leur porter atteinte. (p. 5).

Rodovicz se range du côté de ceux qui plaident pour l'étude de textes entiers. Son premier argument est l'analogie de la classe de français avec celles de latin et d'allemand : aucun professeur de ces matières ne proposerait aux élèves des grandes classes des morceaux choisis. Or son argument le plus fort en est un autre : on n'apercevra jamais l'ensemble d'une œuvre si on se limite à la vue des détails. C'est donc d'une part l'exemple d'autres matières, et notamment celui des langues classiques, mais d'autre part la finalité de la classe de littérature - faire voir aux élèves la valeur, la structure, la beauté d'une œuvre littéraire - qui explique la prise de position en faveur de l'étude d'œuvres entières.

Neubauer (1860) refuse également l'emploi d'anthologies, du moins dans les classes terminales :

(...) un des buts principaux de la lecture française en première, c'est de former, de corriger, d'ennoblir les expressions dont l'élève se sert en écrivant et en parlant. Il faut donc qu'il fasse attention au style de son auteur ; ce qu'il ne peut faire, s'il en lit un autre tous les quinze jours, c'est-à-dire, s'il n'en lit aucun, mais une chrestomathie. (p. 9).

9 Ceci ne l'empêche cependant pas d'admettre l'emploi d'une anthologie en seconde. Là il s'agit

d'apercevoir l'existence, dans les écrivains, des règles de grammaire (...), de se convaincre par l'apparence, c'est-à-dire la lecture, que la grammaire n'existe pas seulement dans les têtes des savants. Telle est la tâche de l'écolier de deuxième, qui n'a pas encore besoin de s'apercevoir ni de s'occuper du style, et qui a fait tout ce qu'on peut désirer, si la lecture l'a complètement fortifié contre les fautes d'étymologie, et passablement contre celles de la syntaxe. C'est pourquoi la chrestomathie s'emploie bien en deuxième. (p. 9). 
Plus l'on avance dans le temps, plus les auteurs se prononcent en faveur d'une nette distribution des tâches entre la chrestomathie et les textes littéraires entiers. Ainsi Timme (1882) plaide pour l'abandon de la chrestomathie à partir de la cinquième année. Pendant les dernières années, on étudiera des textes entiers, et ceci parce que l'on veut que l'élevé plus âgé établisse " une relation personnelle » avec les auteurs du programme et qu'il découvre leur style propre et incomparable. Comment l'élève pourrait-il s'en apercevoir, comment pourrait-il en profiter, s'il ne lui restait pas le temps de s'y introduire par une lecture plus étendue et plus approfondie?

11 Les débats sur « la chrestomathie ou les auteurs » ont eu des répercussions dans les programmes officiels. Ainsi, les programmes prussiens pour les collèges (Realgymnasium / Oberrealschule) de 1882 demandent que l'on abandonne " le plus tôt possible " les chrestomathies en faveur " de la lecture d'œuvres entières qui correspondent quant au contenu et à la forme à l'état d'esprit des classes respectives ${ }^{1}$. Les programmes consacrent ainsi une tendance qui résulte de trente ans de discussions.

\section{En quelle langue enseigner la littérature française?}

notre corpus, nous trouvons des articles rédigés en allemand et en français. ${ }^{2}$ Parm les textes rédigés en français, il y en a quelques-uns qui poursuivent apparemment le but de servir de modèle pour la classe de littérature en français, tout d'abord à l'usage des professeurs. Mais en plus, nous trouvons des textes pour la main de l'élève, également en français, comme par exemple un Abrégé de la littérature française de Fritzmann (1877) ou un Précis de l'histoire de la littérature française de Pétri (1852/ 1854), qui devaient être utilisés pour la préparation des élèves soit au travail en classe, soit au baccalauréat. Il y en avait d'autres rédigés en allemand. Enfin, on trouve de nombreuses « analyses » et " explications » de textes d'auteurs, rédigées en général en français (Fogler 1875 ; Metger 1871), où l'on se demande si elles étaient destinées aux professeurs ou aux élèves. Ils servaient vraisemblablement aux uns comme aux autres.

De toute façon, le choix de la langue est un problème de taille. Mais il est rarement discuté explicitement. Ceci peut étonner en pleine période de la " réforme ». Les tenants de la " réforme " se battaient pour l'emploi de la langue-cible en classe de langue ; or ils se sont intéressés tout d'abord aux classes débutantes et se désintéressaient en partie de la littérature.

Le débat sur le choix de la langue a pourtant trouvé ses répercussions dans certains programmes officiels. Indiquons à titre d'exemple le programme pour les lycées du grand-duché de Bade de 1863, pour les collèges du même pays de 1868, pour les lycées de Bade de 1869, pour les collèges du Royaume de Saxe de 1884, pour les lycées du Royaume de Wurttemberg de $1891^{3}$. Dans ces programmes officiels, il est recommandé aux professeurs de s'exprimer, notamment dans les classes supérieures, en général en français et d'exiger des élèves d'en faire autant. ${ }^{4}$

Il est intéressant d'étudier, sous cet angle, le Questionnaire sur la vie et les cuvres de Molière de Suess (1892) et l'Entretien littéraire entre les aspirants à l'examen de maturité d'Osterberger (1864). Le Questionnaire et l'Entretien ont ceci en commun qu'ils simulent le discours de la classe de littérature. Dans les deux cas, il s'agit de leçons de révision sur ce que l'on a étudié précédemment en classe ou à la maison. 

rôle grandissant, qui doivent (ou veulent) faire leur classe en français, en « dialogue " permanent entre professeurs et élèves. Or le Questionnaire propose une " conversation " spéciale : celle de l'examen. Il est très détaillé : il traite de la vie de Molière, de sa famille, sa jeunesse et sa vocation théâtrale, ses itinéraires, ses troupes, ses bienfaiteurs, ses adversaires, ses pièces, mais aussi de l'histoire sociale et politique de l'époque. L'auteur sait que les professeurs ne peuvent pas traiter tout en détail ; il veut offrir un large choix afin que chaque professeur choisisse ce qui convient. ${ }^{5}$ répondre continuellement en "phrases entières »:

-Quand Molière est-il né ?

-Le 15 janvier 1622.

-Où?

-A Paris. démolition de la salle du Petit-Bourbon et avant la réouverture de la salle du PalaisRoyal, à la recherche d'une salle appropriée aux besoins de sa troupe :

-Où Molière donna-t-il ses représentations pendant ces travaux?

-Il jouait plusieurs fois à la ville, chez plusieurs grands personnages de la cour et de la finance.

-Chez qui, par exemple?

-Chez Fouquet.

-Qui est-ce?

-C'est le fameux surintendant des finances.

-Par quoi est-il notamment connu?

-Par la chute soudaine où il tomba plus tard.

Osterberger - l'auteur de l'Entretien littéraire - partage certaines opinions de Suess. Son Entretien commence sans introduction aucune. Six élèves de la classe terminale se sont rassemblés pour réviser ce qu'ils ont appris en classe de français pendant quatre ans. Louis propose de réviser la grammaire, mais Frédéric préférerait une révision de la littérature : «Un petit entretien touchant la littérature française nous sera plus nécessaire » (p. 3). Et puisque les autres se rangent de son côté, on décide de revoir - en six sessions - tout ce que l'on a appris en littérature. Les élèves assurent la présidence à tour de rôle.

21 L'Entretien, bien que fictif, permet de jeter un regard dans la classe de M.Osterberger. On peut supposer que le texte résume le contenu de ses cours de littérature française et d'histoire littéraire :

-Louis : Eh bien, mon Eugène, le pays qu'on nomme de nos jours la France, portait-il toujours ce même nom?

-Eugène : Point du tout, Monsieur, dans l'Antiquité la France portait le nom de Gaule...

Il suit un bref regard sur le développement de la langue française qui mène aussitôt à la littérature médiévale. "On peut diviser en trois grandes classes ou trois cycles principaux les 
productions chevaleresques de cette période; ce sont les romans de Charlemagne, les romans de la Table ronde et les Romans appelés mixtes. » (p.6). Ceci se montre jusque dans les programmes officiels. Les programmes prussiens étaient très clairs :

De même qu'on ne traite pas, dans les lycées, l'histoire de la littérature romaine ou grecque, on ne traitera pas, dans les collèges, l'histoire de la littérature française ou anglaise comme disciplines à part ; on n'introduira pas de manuel et on ne dictera pas non plus l'histoire littéraire dans un cahier; il suffira de donner les informations nécessaires concernant l'histoire au cours de la lecture, tout comme on les donne d'habitude dans les chrestomathies. Si l'on fait abstraction d'un tel contexte concret, l'acquisition d'un savoir en histoire littéraire séduira l'élève à se vanter de connaissances qu'il n'a pas en réalité ${ }^{6}$ étudiée. On trouve une position identique dans les programmes pour les lycées et 
collèges de filles de Bade de 1892 ("Toute information sur l'histoire littéraire se fera à la suite de la lecture des textes, et on Réutilisera en aucun cas des précis ou des manuels de l'histoire littéraire qu'ils soient imprimés ou écrits $»^{7}$ ) et dans les programmes pour les collèges de Hesse : "On n'enseignera pas l'histoire de la littérature française ; le professeur donnera les informations nécessaires au cours de la lecture $»^{8}$

Les programmes du Royaume de Saxe tiennent un autre discours. On peut lire en 1877 : «Et pour terminer, l'élève aura acquis la connaissance de l'histoire de la littérature française $»{ }^{9}$ Les programmes ne précisent pas de quelle manière cette acquisition devrait se faire : par cours magistral, par un précis ou un manuel, par des notes dictées par le professeur? Pour les lycées saxons, les programmes de la même année sont aussi énigmatiques quant aux procédés, mais aussi clairs quant au but : « Un bref survol sur les principales périodes de l'histoire de la littérature française. $»^{10} Q u e l q u e s$ années plus tard, on lira qu'il faudra traiter dans les lycées « l'essentiel de l'histoire littéraire ». ${ }^{11}$ Pour la classe terminale du collège, les programmes du Royaume de Bavière exigent : "Précis de l'histoire de la littérature française, surtout du XVII et du XVIII siècles ". ${ }^{12}$

Quoi qu'en disent les programmes officiels, la pratique de nombreux professeurs était sans doute celle d'enseigner systématiquement l'histoire littéraire. Nous avons vu que Suess et Osterberger l'ont fait. Dans notre corpus de Schulprogramme se trouvent une dizaine de " précis ", « coup d'œil », « survols » conçus pour la main de l'élève, comme par exemple un Précis de l'histoire de la littérature française de Pétri (1852 et 1854), le Kurzer Abri/3 der französischen Literatur de Schroeter (1853 et 1856), le Coup d'œil sur le théâtre en France de Kantorowicz (1881), un Abrégé de la littérature française, composé à l'usage des classes supérieures des lycées de Fritzmann (1877) et d'autres qui se limitent dans le temps, comme le Coup d'œil général sur la littérature française au dix-neuvième siècle de Robert (1856), ou les Etudes sur la littérature française de Rohde (1879) ou enfin le Coup d'œil sur l'origine et le développement de la langue et la littérature françaises dans la première période de Bourier (1860).

31 Nous étudierons de plus près le Leitfaden der französischen Literaturgeschichte zunächst für die Prima eines Realgymnasiums (Eléments d'histoire de la littérature française pour les deux dernières classes d'un collège) de Herford (1886). L'auteur a conçu son Leitfaden pour ses propres élèves, mais il le publie dans l'espoir que d'autres en profitent. ${ }^{13}$ L'étude de l'histoire de la littérature française a une double fonction : préparer les élèves des grandes classes à - comme on dirait de nos jours l'apprentissage tout au long de la vie ${ }^{14}$ et de servir de cadre pour intégrer les textes étudiés en classe, qui se présentent aux élèves comme des cas isolés. ${ }^{15}$

32 Le Leitfaden se compose d'une introduction, de trois grands chapitres et d'un épilogue. Le premier chapitre traite du développement de la langue française jusqu'à l'époque moderne. Le second chapitre s'occupe de la littérature épique et dramatique du Moyen Age et de la Renaissance. Le troisième chapitre est le plus long et se voue essentiellement au théâtre du XVII ${ }^{e}$ siècle, avec - en appendice - quelques remarques sur La Fontaine et Bossuet. Suivent en épilogue la littérature du XVIII ${ }^{\mathrm{e}}$ et du XIX ${ }^{\mathrm{e}}$ siècles.

33 Prenons à titre d'exemple les pages qui traitent de Racine (p. XII-XIV). Après une introduction biographique en quelques lignes, l'auteur présente Andromaque, Britannicus, Mithridate, Iphigénie, Phèdre et Athalie. Il positionne les pièces dans le temps, fait quelques remarques sur leur succès auprès du public et ne retient pas son propre jugement (par exemple «Athalie (1691), le chant du cygne de Racine, le chef- 
d'œuvre des tragédies françaises $\gg{ }^{16}$ ) L'essentiel de ces présentations, ce sont les résumés et les analyses des pièces. Comme Racine, les deux autres grands dramaturges classiques sont traités de la même manière. Or, pour le reste de la littérature française, et notamment pour le XIX ${ }^{\mathrm{e}}$ siècle, Herford perd le souffle. Victor Hugo a juste droit à huit lignes, Scribe et George Sand à deux et pour Alfred de Musset, Alfred de Vigny et Alexandre Dumas il suffit d'une seule.

Or beaucoup de professeurs ont refusé ce type de leçons et se sont contentés de remarques historiques préliminaires et corollaires à la lecture, comme la plupart des programmes officiels le demandaient et comme de nombreuses " lectures » à l'usage des élèves ou de chrestomathies ou de collections de poèmes le suggéraient. Je cite à titre d'exemple la Sammlung französischer Gedichte de Truemper (1894). Sa collection propose une quarantaine de poèmes de La Fontaine à François Coppée, accompagnés d'un Précis de versification, de remarques d'histoire littéraire et d'aides au travail à la maison. Elle est destinée aux troisièmes et secondes.

Je cite - en traduisant - une de ces « remarques d'histoire littéraire » :

Béranger, Pierre-Jean de, 1780-1857, raconte l'histoire de sa jeunesse dans les deux premières strophes de 'Le Tailleur et la Fée'. Il fréquentait une école républicaine à Péronne et étudiait Racine, Fénelon et Voltaire; notamment le dernier exerçait une grande influence sur lui. A l'âge de 16 ans, il retournait à Paris et travaillait dans la banque de son père qui pourtant n'était pas riche. Bientôt son génie poétique s'éveillait. Ses chansons émerveillaient tout le monde et ses chants populaires avaient une profonde signification politique. Il haïssait les Bourbons, s'enthousiasmait malgré ses penchants républicains pour Napoléon et avait un amour touchant de l'indépendance et de la simplicité. Il était un Français insouciant et malheureusement aussi frivole, un poète populaire au sens plein du mot, et il en était fier. Le meilleur chansonnier de France, ceci est sa grandeur. ${ }^{17}$

\section{Comment présenter et traiter des textes littéraires en classe?}

Nous en venons enfin à la question méthodologique par excellence, à savoir l'agencement et le déroulement de la classe de littérature. Quelle a été la pratique quotidienne ? On en parle relativement peu dans les articles de notre corpus, et si on le fait, on se contente très souvent - à quelques exceptions près - de remarques très sommaires.

Il est à supposer qu'il y avait des traditions qui émanent de l'enseignement des langues classiques. C'est ce que constate Ulbrich (1884) qui mentionne trois moments de la classe de littérature, se basant sur cette tradition : lire, traduire et expliquer (sans d'ailleurs dire qui lit, qui traduit, qui explique). "Ce que l'on reproche à cette méthode de plusieurs côtés, c'est l'ordre de ces trois opérations, de la lecture, de la traduction et de l'explication " gui devrait - à son avis - être renversé : à la lecture (à haute voix) reviendrait la dernière place, elle devrait être considérée comme le résultat de la classe. ${ }^{18}$ Muench (1979) souligne, également, que la classe de langue vivante suit « un bout de chemin » les méthodes de l'enseignement des langues classiques ; mais il réclame qu'elle n'oublie pas ses buts spécifiques:

"Sans faire des concessions non justifiées aux seuls buts pratiques, on n'oubliera pas que nous visons - à côté de la pénétration logique du texte - une certaine maîtrise de la langue et que nous cultivons à côté du savoir avant tout le savoir-faire langagier. $»^{19}$ 
Steinmueller (1893) expose dans la préface d'une petite anthologie poétique comment il procède en classe. Il distingue cinq moments de la classe de littérature :

« $1^{0}$ On lira et traduira dans un premier pas le poème en petites fractions $» .^{20} \mathrm{Il}$ ne dit pas qui est « on » : Est-ce le professeur ou sont-ce les élèves ? Quelle est la fonction de la traduction? Il s'agit, paraît-il, d'une « version de travail » qui sert à garantir la bonne compréhension des détails du texte par la classe.

« $2^{\circ} C^{\prime}$ est d'abord le professeur, ensuite ce sont les élèves qui liront le texte dans son ensemble ${ }^{21}$ La lecture se fait donc avant l'explication du texte. On peut supposer que cette « lecture » (en allemand « Vorlesen » et non pas « Lesen ») frise la « diction ».

" $3^{\circ}$ Le professeur pose des questions en langue française pour expliquer le texte $» .^{22}$ Tandis que pendant les deux premiers moments de la classe on se sert de l'allemand pour traduire le texte et pour corriger des fautes de prononciation et de diction, ce troisième moment se passe en français. Le professeur pose des questions (Steinmueller en donne quelques exemples à l'appendice) et les élèves répondent. C'est donc à eux qu'incombe le travail de l'explication, évidemment dirigés et aidés par le professeur. " $4^{\circ}$ On résumera le poème en prose $» .{ }^{23}$ Le quatrième moment se passe également en français et il est à supposer qu'il est essentiellement l'affaire des élèves. Il est évident que le résumé ou (comme dit Steinmueller) la « narration » différera considérablement selon le poème en question. « $5^{\circ}$ On mémorisera le poème éventuellement $\gg .{ }^{24}$ Cette tâche se fera sans doute à la maison.

2 Nous citerons encore un autre auteur pour montrer que la méthode proposée par Steinmueller correspondait - dans les grandes lignes - à ce que de nombreux professeurs pratiquaient. Ulbrich (1889) écrit :

La façon dans laquelle on traite la lecture de textes littéraires actuellement, est pour le français (...) de la sixième jusqu'à la terminale à peu de différences près en général la suivante : un élève lit un paragraphe d'un texte, le traduit avec l'aide du professeur ou d'un autre élève destiné à cette fin ; après ceci, on reprend le texte mot par mot, pour garantir la compréhension, et on explique les constructions des phrases (...) Dans la leçon suivante, on répète en général la traduction (...)..$^{25}$

43 La différence essentielle entre Ulbrich et Steinmueller est le fait que Steinmueller parle explicitement des grandes classes, tandis qu'Ulbrich prétend qu'il y a un consensus pour pratiquement toutes les classes du lycée et du collège.

Il paraît que le premier moment de la classe de littérature dans les grandes classes - à savoir lire un texte pas à pas et le traduire - a été accepté tel quel par la majorité des professeurs. Vogel (1880 : 18) propose cependant que l'explication se fasse simultanément, notamment au début d'une lecture suivie, et ceci lentement et de façon détaillée. Pour lui, c'est la tâche du professeur de donner les explications. Quant à l'élève, il sera actif à la maison. Il se préparera à traduire le texte lu en classe dans la leçon suivante "couramment et en bon allemand ". ${ }^{26}$ Après avoir traité une partie convenable du texte, "le professeur interrogera les élèves sur l'acquis langagier (das Sprachmaterial) et sur le contenu du texte " afin que l'élève assimile vraiment et le contenu et les éléments langagiers, et dans la même intention il leur donnera différents devoirs comme de brefs exposés oraux, éventuellement aussi des essais à faire à la maison. Au cours de l'année, il renoncera aux traductions et les élèves prépareront au lieu de cela des résumés écrits ou oraux en français.

Voyons cependant ce que l'on comprend par " explication ». Nous citons à titre d'exemple "L'explication d'un drame français » de Metger (1871). Ce n'est pas la pièce 
traitée qui est intéressante - une comédie historique de M. J. N. Bouilly, L'Abbé de l'Epée mais ce sont les idées méthodologiques du professeur Metger:

Je le crois nécessaire de développer en peu de mots les principes d'où je pars et la méthode que je suis, pour faire tirer aux élèves le plus de profit possible de cette lecture. Il s'agit d'abord de ce que l'on appelle l'économie d'une pièce de théâtre, c'est-à-dire le plan de l'ouvrage, de la marche des idées, du développement des caractères, de la suite des situations, etc. Mais étant l'affaire du maître d'expliquer tout cela de vive voix, il ne me paraît pas convenable de donner lieu à ces renseignements dans un écrit destiné à l'usage des élèves (p. 3).

Secret professionnel oblige! Ainsi nous n'apprenons pas comment le professeur a conçu ses leçons magistrales:

En second lieu je trouve dans cette lecture la meilleure occasion d'exercer les jeunes gens à parler français, parce que la plupart de ces pièces sont composées dans le langage de la vie ordinaire. C'est donc en apprenant par cour une partie des passages traduits et analysés qu'on s'approprie nombre d'expressions et de phrases, et qu'on gagne par là cette volubilité de la langue si nécessaire pour bien s'énoncer (p. 3).

Nous apprenons un point essentiel pour le choix de la pièce expliquée : elle est écrite dans un langage " de la vie ordinaire ». Après avoir appris par cœur des passages de la pièce, les élèves disposeront d'un stock de locutions, d'expressions et de phrases toutes faites qui leur serviront dans la conversation.

Mais je crois avoir trouvé encore un autre moyen d'accoutumer les élèves à s'y appliquer. $C^{\prime}$ est celui de faire donner des définitions. C'est la définition qui non seulement engage l'élève $\grave{a}$ se familiariser avec les diverses expressions pour la même chose, comme avec les différentes acceptions des mots, mais qui aussi l'accoutume à se procurer cet art de définir, $c$ 'est-à-dire d'attacher aux mots des idées justes et précises, qui de tout temps a été considéré comme la chose la plus difficile (p. 3-4).

L'explication du drame consiste effectivement en grande partie en de telles définitions qui sont données en bas de la page. Voilà une de ces définitions : «Acte : l'une des parties principales dans lesquelles se divise une pièce de théâtre » (p. 7). Nous imaginons le travail en classe : ou le professeur donne les explications, souvent en forme de définitions et de vive voix, et les élèves les répètent, ou les élèves trouvent les explications eux-mêmes en bas de la page, les déchiffrent et les emploient, interrogés par le professeur ou dans leur préparation à la maison. Voilà donc comment l'enseignement de la langue et de la littérature sont méthodiquement liés.

Après avoir traité du problème de l'explication, il nous reste à parler de la traduction exercée dans les leçons de littérature. Ulbrich (1884) est assez critique à son égard : "Ce que l'on exerce ici (sc. dans la classe de littérature) c'est la bonne version du texte étranger en langue maternelle (...). Est-ce le but principal d'un enseignement de français pendant huit ans? Ne serait-ce pas plutôt l'expression orale et écrite? $»^{27}$

La version a trouvé d'autres critiques plus sévères. On sait par exemple que Viëtor, l'auteur du fameux manifeste publié sous le pseudonyme de "Quousque tandem» (1882), était un adversaire de la version par principe: «La version est un art qui $n$ 'a rien à voir dans la classe de langue étrangère ». L. von Pfeil (1883) - un autre adversaire de la version - disait que toute version soignée, donc en "bon allemand ", détournerait les pensées des élèves de la langue étrangère. ${ }^{28}$

51 Il y avait donc deux arguments de poids contre la pratique de la version dans la classe de littérature : la version soignée vise trop haut, et elle détourne l'élève de ce qu'il devrait faire, lire des textes en langue étrangère et employer cette langue. Mais il ne 
manquait pas d'autres voix qui défendaient la version, encore en pleine période de la " réforme ". Nous trouvons dans notre corpus Lobedanz (1890) comme un de ses défenseurs. Il souligne l'effet formateur de la version (" den bildenden Einfluss ») ; il refuse la version approximative qu'il qualifie de « mauvaise traduction », il pense que la bonne version n'améliore pas seulement la langue maternelle de l'élève, mais qu'elle lui donne une idée générale du fonctionnement des langues. C'est pourquoi il considère qu'il faudrait commencer dans les classes inférieures à enseigner - à côté de la version mot à mot-« la version libre ». ${ }^{29}$

Muench (1886), autre défenseur de la version en classe de langue vivante, voit dans la version un moyen excellent pour développer la langue maternelle : « Je pense que les élèves apprennent leur allemand pour la plus grande partie en traduisant des auteurs étrangers $»{ }^{30}$

Pour Ruehlmann (1891) enfin, la version est le but et le centre de la lecture de textes littéraires, parce qu'elle donne l'occasion de faire des études de grammaire comparée. Ainsi on peut montrer aux élèves comment on exprime en allemand la différence entre l'imparfait et le passé simple ou comment on peut traduire une expression verbale par une expression nominale ou vice-versa.

Bref : la version, qui avait - dans une forme ou dans l'autre - une position forte dans la classe de littérature, n'était pourtant pas acceptée à l'unanimité. Ceux qui la pratiquaient le faisaient avec des finalités très différentes. Ceux qui voulaient la bannir acceptaient malgré tout la version comme moyen de contrôle de la bonne compréhension.

\section{En guise de conclusion}

Nous avons essayé de retracer, dans cet article, de façon incomplète, le cheminement de la pensée méthodologique des professeurs de français dans les pays de langue allemande dans la deuxième moitié du XIXe siècle, pour la classe de littérature. Ce faisant, nous avons repris des études antérieures concernant le choix des textes littéraires dans la classe de français en Allemagne dans la même époque (Christ 1990). Après cette nouvelle approche, nous ne sommes pas arrivés à la fin de notre recherche. Il manque encore d'autres dimensions qui permettront de compléter l'image de l'enseignement de la littérature française : la dimension des apprenants, la dimension des collections de textes et des manuels, la dimension de la réception de la littérature française dans le grand public grâce à l'action de l'école. Autant de travaux à faire...

\section{BIBLIOGRAPHY}

Ouvrages étudiés 
BOURIER, Félix (1860), Coup d'œil sur l'origine et le développement de la langue et de la littérature françaises dans la première période suivi de quelques observations sur l'enseignement de cette langue. Augsburg, Gewerbeschule.

FOGLER, Benedict (1875), Analyse d'Athalie de Jean Racine. Brunn, Staats-Oberrealschule.

FRITZMANN, André (1877), Abrégé de la littérature française, composé à l'usage des classes supérieures des lycées, Bensheim, Gymnasium 1877.

HERFORD, Eugen (1886), Leitfaden der französischen Literaturgeschichte, zunächst für die Prima eines Realgymnasiums, Thorn, Gymnasium (Beilage).

KANTOROWICZ, Camillo (1881), Coup d'œil sur le théâtre en France depuis son origine jusqu 'à la révolution de 1789. Zurich, Kantonsschule.

LOBEDANZ, Emil (1890),Über den Unterricht in Lektüre und Grammatik, besonders im Französischen, Schwerin, Realgymnasium (Beilage).

METGER, Heinrich (1871), Explication d'un drame français. Verden, Gymnasium.

MUENCH, Wilhelm (1879), Bemerkungen über die französische und englische Lektüre in den oberen Realclassen, Ruhrort, Realschule.

MUENCH, Wilhelm (1886), « Die Kunst des Übersetzens aus dem Französische », in : Zeitschrift fiir neufranzösische Sprache und Litteratur IX.

NEUBAUER, Heinrich (1860), La chrestomathie ou l'auteur? Questions de philologie moderne, Halle/Saale, Realschule.

OSTERBERGER, Bonifaz (1864), Entretien littéraire entre les aspirants à l'examen de maturité Münnerstadt, Gymnasium (Beilage).

PÉTRI, Ulrich $(1852,1854)$, Précis de l'histoire de la littérature française. Première et deuxième parties. Barmen, Gymnasium.

Quousque tandem (i. e. Wilhelm Viëtor) (1882), Der Sprachunterricht muss umkehren. Heilbronn 1882.

ROBERT, Jules Vivant (1856), Coup d'œil général sur la littérature française au dix-neuvième siècle. Rostock, Gymnasium.

RoDowicz, G. (1852), De quelle façon pourrait-on avantageusement modifier l'étude de la littérature française dans nos collèges? Krotoschin, Realschule.

ROHDE, Theodor (1879), Etudes sur la littérature française. Première étude : Le XVII siècle Pillau, Hohere Bürgerschule.

RUEHLMANN, Otto (1891), Zur Behandlung der Lektüre im Französischen. Halle/Saale, Realgymnasium. SCHROETER $(1853,1856)$, Kurzer Abriss der französischen Literatur (Fortsetzung und Schlufi) Lübben, Höhere Burgerschule.

SUESS, Heinrich (1892), Questionnaire sur la vie et les œuvres de Molière. Introduction à la lecture de ce poète, d'après l'édition des cuvres complètes de Molière par Moland. Partie première. Strehlen, Gymnasium (Beilage).

TIMME Otto (1882), Über die Auswahl von französischer Lektüre für die oberen Realklassen. Hildesheim, Gymnasium (Beilage). 
TRUEMPER, Karl (1894), Sammlung französischer Gedichte nebst kurzgefasster Verslehre, litteraturgeschichtlicher Bemerkungen und Hilfe für die häusliche Vorbereitung (I. Teil, besonders für Tertia und Secunda). Duderstadt, Gymnasium (Beilage).

ULBRICH, Oskar (1884), Über die französische Lektüre an Realgymnasien. Berlin, Friedrichs-

Realgymnasium (Beilage).

Lekture in den oberen Realschulklassen. Perleberg, Realschule.

\section{Ouvrages consultés}

CHRIST, Herbert (1990), « Littérature française et enseignement du français au XIX ${ }^{\mathrm{e}}$ siècle dans les lycées et collèges allemands », in : Documents 8, 1990, p. 174-211.

CHRIST, Herbert / Rang, Hans-Joachim (éds.) (1985), Fremdsprachenunterricht unter staatlicher Verwaltung J 700-1945. Eine Dokumentation amtlicher Richtlinien und Verordnungen. 7 tomes, Tübingen.

KÖSSLER, Franz (1987-1991), Verzeichnis von Programm-Abhandlungen deutscher, österreichischer und schweizerischer Schulen der Jahre 1825-1918. 5 tomes, München.

\section{NOTES}

1. «Es ist dabei möglichst bald von dem Gebrauche der Chrestomathien zur Lektüre von ganzen Schriftwerken fortzuschreiten, deren Inhalt und Darstellung dem Standpunkte der einzelnen Klassen entspricht. » (Lehrpläne für die höheren Schulen. 1882, Christ/Rang II, p. 66).

2. Dans le corpus complet (voir Kössler 1987/1991) on trouve aussi des articles rédigés en hongrois, italien, tchèque et polonais que nous n'avons pas pris en considération, vu notre champ de recherche limité : les collèges et lycées de langue allemande.

3. On les trouve tous dans Christ/Rang II et III, 1985.

4. Voilà à titre d'exemple les règlements pour le collège du Grand Duché de Bade de 1868 : «Von der VI. Klasse an (c'est-à-dire pendant les deux dernières années ) soll der Verkehr zwischen Lehrer und Schüler in den französischen Lehrstunden in der Regelin französischer Sprache stattfinden. » (cf. Christ/Rang 1985 III, p. 28).

5. «Zwar wird die Einleitung über das Leben und die Werke Molières in dieser Ausführlichkeit, wie sie die vorliegende Arbeit bietet, wohl kaum durchgenommen werden, aber 'wer vieles bringt, wird manchem etwas bringen' ; es wähle jeder, was er braucht. » (Suess, Vorbemerkung, p. 1).

6. "So wenig auf Gymnasien römische und griechische Literaturgeschichte gelehrt wird, ist auf den Realschulen französische und englische Literaturgeschichte als besondere Disziplin zu behandeln ; es ist dafür weder ein Buch einzuführen, noch ein Heft zu diktiren ; es genügt, die erforderlichen geschichtlichen Mittheilungen an die Lektüre anzuschliessen, wie sie auch in den Chrestomathieen mit der Auswahl der Stiicke verbunden zu sein pflegen. Ohne diesen Zusammenhang verleitet die Aneignung literar-historischer Notizen leicht dazu, mit eingebildeten Kenntnissen zu prunken. » Unterrichts- und Prüfungs-Ordnung der Realschulen und höheren Bürgerschulen, 6 octobre 1859, in : Christ/Rang 1985, II : 63.

7. «Alle litteraturgeschichtliche Belehrung ist an die Lektüre anzuschliessen und es dürfen keinerlei gedruckte oder geschriebene litteraturgeschichtliche Leitfäden oder Lehrbücher gebraucht werden. » Lehrplan der höheren Mädchenschulen im Grossherzogtum Baden, 22 octobre 1892, Christ/Rang III : 62 .

8. " Ein besonderer Unterricht in der französischen Literaturgeschichte wird nicht gegeben, sondem das zum Verständnis des betreffenden Schriftstellers Notwendige bei der Lektüre 
mitgeteilt. » Lehrplan für die Realgymnasien des Grossherzogtums Hessen, 18. 1. 1893, in : Christ/ Rang III : 66.

9. « Endlich muss die Bekanntschaft mit der französischen Literaturgeschichte erworben sein. » Lehr- und Prufungsordnung für die Realschulen I. Ordnung, 29. 1. 1877, in : Christ/Rang III : 4.

10. "Ein kurzer Überblick über die Hauptperioden der französischen Literaturgeschichte. » Lehr- und Prüfungsordnung für die Gymnasien, 29. 1. 1877, in : Christ/Rang III : 32.

11. «Das Wesentliche aus der Litteraturgeschichte. " Lehr- und Prüfungsordnung für die Realgymnasien, 18. 2.1884, in : Christ/Rang III : 41.

12. "Neunte Klasse ; Abriss der französischen Literaturgeschichte, besonders des XVII. und XVIII. Jahrhunderts. » Schulordnung für die Realgymnasien im Königreiche Bayern, 3. 9. 1891, in : Christ/Rang III : 55.

13. « Und so wage ich es denn, diesen lediglich aus der Praxis hervorgegangenen Versuch hiemit zu veröffentlichen, der zunächst für den Gebrauch meiner Schuler bestimmt ist. » (Herford 1886 : III). 14. « die Schüler (...) mit den Mitteln auszurüsten vermöge derer sie nach vollendeter Schulzeit selbständig dièse Kenntnis erwerben beziehentlich erweitern können. » (Herford 1886 : III).

15. " Durch die Lekture lässt sich dièses Ziel doch nur sehr bruchstuckhaft erreichen, die Literaturgeschichte kann also helfend eintreten, namentlich durch Inhaltsangaben und Mitteilung wichtiger Proben. » (Herford 1886 : III).

16. «Athalie (1691), der Schwanengesang Racine's, das Meisterwerk der französischen Tragik. » (Herford 1886 : XIV).

17. « Béranger, Pierre-Jean de, 1780-1857, erzählt seine Jugendgeschichte in der 1. und 2. Strophe von 'Le Tailleur et la Fée'. Er besuchte eine republikanische Schule in Péronne und las viel in Racine, Fénelon, Voltaire ; besonders der letztere ubte groften Einfluss auf ihn. Mit 16 Jahren kehrte er nach Paris zurück und war in dem Bankgeschäfte seines keineswegs reichen Vaters thätig. Bald jedoch erwachte sein dichterisches Génie. Mit seinen chansonsentzückt er aile Welt und seine Liederchen wurden von hochpolitischer Bedeutung. Er hasste die Bourbonen, schwärmte trotz seiner echt republikanischen Gesinnung für Napoléon und hatte eine rührende Liebe zur Unabhängigkeit und Einfachheit. Ein leichtlebiger und leider auch leichtfertiger Franzose ; ein Volksdichter durch und durch, und das war sein Stolz; der vorzüglichste Chansonnier Frankreichs, und das ist seine Grösse » (Trümper 1894 : 64).

18. «Im übrigen ist die Behandlung der Lektüre hier durch die Tradition gegeben, welche ganz der im Latein-Unterricht ublichen Méthode gefolgt ist. Was von mehrereSeiten an ihr getadelt wird, ist die Reihenfolge der dabei notwendigen Operationendes Lesens, Übersetzens, Erklärens, von denen die erste aus sehr naheliegendeGründen die letzte sein müsste. » (Ulbrich 1884 : 29).

19. «Ohne den Ansprüchen des praktischen Bedürfnisses unmotivirte Concessionen zu machen, muss man sagen, dass hier gegenüber bloss logischer Durchdringung ein gewisses Beherrschen der Sprachen Ziel ist, dass gegenüber dem Wissen auch das Können in beträchtlichem Masse gepflegt werden soll. » (Muench $1879: 4$ ).

20. «1. Das Gedicht wird zuerst bruchweise gelesen und übersetzt. » (p. 4).

21. « 2. zuerst vom Lehrer, dann vom Schüler dem Zusammenhang nach vorgelesen. » (M).

22. «3. durch Fragen in französischer Sprache erläutert. » (p. 4).

23. « 4. in Prosa nacherzählt. » (p. 4).

24. «5. (gegebenen Falls) memoriert. » (p. 4).

25. «Die Art, wie jetzt die Lektüre betrieben wird, ist im Französischen (...) von der untersten bis zur obersten Klasse mit geringen Abweichungen gewöhnlich die folgende: ein Schüler liest einen Abschnitt des fremden Textes vor, ubersetzt denselben mit Nachhülfe des Lehrers oder dazu aufgerufener Schüler ; darauf wird derselbe Abschnitt Wort für Wort durchgegangen, das Verständnis gesichert, die Konstruktionen erklärt (...) In der folgenden Stunde wird die (...) Übersetzung gewöhnlich wiederholt. » (Ulbrich $1884: 24)$. 
26. «AlleSchüler haben sich zur folgenden Stunde zur flieBendem in gutem Deutsch vorgetragenen Wiedergabe des ubersetzten Pensums vorzubereiten. » (Vogel $1880: 18$ ).

27. «Was also hier (...) vonehmlich geübt wird, ist die richtige Wiedergabe des fremden Textes durch die Muttersprache (...) Ist dies aber der Hauptzweck des achtjährigen Französischunterrichts ? Soll nicht das Sprechen und das Schreiben (...) daneben auch abfallen ? »(Ulbrich $1884: 24$ ).

28. Beim Übersetzen ist der korrekte Ausdruck in der Muttersprache zu vernachlässigen, denn das Streben nach solchem Ausdruck würde die Gedanken des Lernenden von der Sprache ablenken. » (Pfeil 1883, cité dans Lobedanz $1890: 34$ ).

29. "Schon in den unteren Klassen ist es daher zweckmässig, ausser der wörtlichen eine freie Übertragung zu geben. » (Lobedanz $1890: 34$ ).

30. «Ihr meistes Deutsch, glaube ich, lernen die Schüler durch das Übersetzen au s einem fremden Autor.» (Muench, p. 79).

\section{AUTHOR}

HERBERT CHRIST

Université de Giessen 